\title{
Comparing Cournot and Bertrand Equilibria in a Differentiated Duopoly with Product R\&D
}

\author{
George Symeonidis \\ University of Essex
}

\begin{abstract}
This paper compares Bertrand and Cournot equilibria in a differentiated duopoly with substitute goods and product R\&D. I find that R\&D expenditure, prices and firms' net profits are always higher under quantity competition than under price competition. Furthermore, output, consumer surplus and total welfare are higher in the Bertrand equilibrium than in the Cournot equilibrium if either $R \& D$ spillovers are weak or products are sufficiently differentiated. If $R \& D$ spillovers are strong and products are not too differentiated, then output, consumer surplus and total welfare are lower in the Bertrand case than in the Cournot case. Thus a key finding of the paper is that there are circumstances where quantity competition can be more beneficial than price competition both for consumers and for firms.
\end{abstract}

Keywords: Product R\&D, price versus quantity competition, welfare.

JEL classification: L13, D43.

Address for correspondence: Department of Economics, University of Essex, Wivenhoe Park, Colchester CO4 3SQ, U.K.

Phone: +44 1206 872511, fax: +44 1206 872724, e-mail: symeonid@essex.ac.uk 


\section{Introduction.}

The standard view that Bertrand competition is more efficient than Cournot competition has recently been challenged by a number of theoretical models. A common feature of these models is the idea that firms compete both in variables that can be easily changed in the short run, such as price or output, and in variables that constitute longer-term commitments, such as capacity or R\&D expenditure. Much of this literature has specifically focused on expenditure in process $R \& D$ as the long-run decision variable. Product $R \& D$, although empirically more important (see Scherer and Ross 1990), has received little attention in these studies.

This paper compares Bertrand and Cournot equilibria in a differentiated duopoly with substitute goods and product R\&D. Vives (1985) and Singh and Vives (1984) found that Bertrand competition results in higher consumer surplus, lower profits and higher overall welfare than Cournot competition in a duopoly model where goods are substitutes and the firms' only choice variable is either price or output. Motta (1993) found price competition to result in higher consumer surplus, profits and overall welfare than quantity competition in the context of a vertically differentiated duopoly with either fixed or variable costs of quality improvement. Although the case of fixed costs of quality improvement can be naturally interpreted as product $R \& D$, the study by Motta did not allow for R\&D spillovers. On the other hand, Delbono and Denicolo (1990) found that the welfare comparison between Bertrand and Cournot is generally ambiguous in the context of a homogeneous product duopoly with process $R \& D$ in the form of a patent race, although $R \& D$ investment is higher in the Bertrand equilibrium (in fact, it is even higher than the socially optimal level). Finally, Qiu (1997), who used the Singh and Vives (1984) linear demand structure but introduced a stage of process $\mathrm{R} \& \mathrm{D}$ competition prior to the price or quantity-setting stage, found 
that $\mathrm{R} \& \mathrm{D}$ expenditure is higher under Cournot than under Bertrand; that the opposite is true for consumer surplus; that the Bertrand equilibrium is more efficient than the Cournot equilibrium if either R\&D productivity is low, or spillovers are weak, or products are very differentiated; and that the Bertrand equilibrium is less efficient than the Cournot equilibrium if $R \& D$ productivity is high, spillovers are strong, and products are close substitutes.

This paper addresses these issues in the context of a model with both horizontal and vertical product differentiation, the latter of which is due to product R\&D. An important difference between process and product $R \& D$ is that the latter directly affects gross consumer surplus. This is because product $R \& D$ raises product quality, and quality enters directly into each consumer's utility function. On the other hand, process R\&D affects gross consumer surplus only indirectly, through a reduction in marginal cost and a consequent increase in output. Thus it is not clear whether the results from models with process R\&D carry over to the case of product R\&D. Moreover, the present model differs from models of 'pure vertical differentiation', such as the one used by Motta (1993), in important ways, including key properties of the firms' profit functions, as will be explained in the next section. It is therefore also an open question whether the existing results from the literature on vertical product differentiation are robust to alternative ways of introducing quality into the utility function.

\footnotetext{
${ }^{1}$ Bester and Petrakis (1993), who also analysed a game of investment in process R\&D using a linear demand structure, obtained results different from those of Qiu (1997) because in their model only one firm was allowed to invest in R\&D.
} 
I analyse here a variant of the standard linear demand model. In particular, I use the quality-augmented linear demand structure proposed by Sutton $(1997,1998)$, and I also introduce a cost structure that allows for R\&D spillovers. I also check the robustness of my results to alternative demand systems and R\&D cost functions. I find that $R \& D$ expenditure, prices and firms' net profits are always higher under quantity competition than under price competition. Furthermore, output, consumer surplus and total welfare are higher in the Bertrand equilibrium than in the Cournot equilibrium if either $R \& D$ spillovers are weak or products are sufficiently differentiated. If $\mathrm{R} \& \mathrm{D}$ spillovers are strong and products are not too differentiated, then output, consumer surplus and total welfare are lower in the Bertrand case than in the Cournot case.

Some of these results are consistent with those obtained by Qiu (1997) in the context of a model with process R\&D. There are also, however, important differences. Under process $R \& D$, consumer surplus is always higher under Bertrand competition, just as in Singh and Vives (1984), but the overall efficiency comparison of the two regimes is ambiguous, presumably because Cournot profits can be a lot higher than Bertrand profits, thus causing the ambiguity. On the other hand, in the present model of product $\mathrm{R} \& \mathrm{D}$, the overall welfare comparison is also ambiguous, but for different reasons. In particular, not only profits but also consumer surplus can be higher in the Cournot equilibrium; this will occur if both R\&D spillovers are strong and the goods are not too differentiated. This is a new result and suggests that both consumers and producers can be better off under quantity competition than under price competition. 


\section{The Model.}

Consider a duopoly where each firm produces one variety of a differentiated product. Firms invest in R\&D in order to enhance product quality. More specifically, competition in the industry is described by a two-stage game as follows. At stage 1 each firm chooses a variety, described by a vertical attribute $u$ that I will call 'quality' and is associated with some physical product characteristic. Quality increases the consumers' willingness to pay for the firm's variety, but it comes at a cost, namely expenditure on product R\&D. At stage 2 the firms set quantities or prices.

The structure of this game reflects the fact that (i) decisions on $R \& D$ expenditure involve significant sunk costs and (ii) product characteristics cannot be changed as easily or as quickly as price or quantity choices. However, I abstract from issues of uncertainty regarding the outcome of $R \& D$ projects, as do many models within the general class of 'non-tournament' models of R\&D rivalry, of which the present model is an example. The key underlying assumption of these models, as opposed to the literature on patent races, is that each firm can achieve product or process improvements through its $R \& D$ expenditure without preventing other firms from obtaining equivalent benefits from their own R\&D.

There are $S$ identical consumers, and the utility function of each consumer takes the form

$U=x_{i}+x_{j}-\frac{x_{i}^{2}}{u_{i}^{2}}-\frac{x_{j}^{2}}{u_{j}^{2}}-\sigma \frac{x_{i}}{u_{i}} \frac{x_{j}}{u_{j}}+M$,

following Sutton $(1997,1998)$ (see also Symeonidis 1999, 2000). Note that (1) is a quality-augmented version of the standard quadratic utility function used, among others, by Singh and Vives (1984), Vives (1985), and Qiu (1997). Thus $x_{i}$ and $u_{i}$ are, respectively, the quantity and quality of variety $i$ and $M=Y-p_{i} x_{i}-p_{j} x_{j}$ denotes 
expenditure on outside goods. This utility function implies that each consumer spends only a small part of her income on the industry's product (which also ensures that the maximisation of $U$ has an interior solution) and hence income effects on the industry under consideration can be ignored and partial equilibrium analysis can be applied. The parameter $\sigma, \sigma \in(0,2)$, is an (exogenous) inverse measure of the degree of horizontal product differentiation: in the limit as $\sigma \rightarrow 0$ the goods become independent, while in the limit as $\sigma \rightarrow 2$ they become perfect substitutes when $u_{i}=u_{j}$.

The inverse demand function of each consumer for variety $i$ is given by

$$
p_{i}=1-\frac{2 x_{i}}{u_{i}^{2}}-\frac{\sigma}{u_{i}} \frac{x_{j}}{u_{j}}, \quad i, j=1,2, \quad i \neq j
$$

in the region of quantity spaces where prices are positive. The demand function is

$$
\frac{x_{i}}{u_{i}}=\frac{2 u_{i}\left(1-p_{i}\right)-\sigma u_{j}\left(1-p_{j}\right)}{(2-\sigma)(2+\sigma)}, \quad i, j=1,2, \quad i \neq j,
$$

in the region of prices where quantities are positive. It can be easily seen that $x_{i}$ is decreasing in $p_{i}$ and increasing in $u_{i}$. Also, it is increasing in $p_{j}$ and decreasing in $u_{j}$. An attractive feature of the present model - and one that distinguishes it from models of 'pure vertical differentiation', which do not have this property - is that it gives rise to reduced-form profit functions where (gross) profit increases in own quality and decreases in rival quality (see below). Since there are $S$ consumers, firm $i$ sells quantity $S x_{i}$. Let each firm have a constant marginal cost of production $c$, where $c<1$. Admittedly, the demand system used in this paper is rather specific. To check the robustness of my results with respect to other specifications of demand, I analyse in the appendix an alternative extension of the quadratic utility model, following Häckner (2000). The results from this alternative demand system are very similar to 
those presented in the main body of the paper: all the propositions proved below are robust to the choice of demand specification.

The function linking quality $u_{i}$ to $\mathrm{R} \& \mathrm{D}$ expenditures $R_{i}$ and $R_{j}$ is a modified version of the one used by Motta (1992) and is given by $u_{i}=\varepsilon R_{i}^{1 / 4}+\varepsilon \rho R_{j}^{1 / 4}$, where $\varepsilon, \varepsilon$ $>0$, is an inverse measure of the cost of $\mathrm{R} \& \mathrm{D}$ or an index of technological opportunity in the industry and $\rho, \rho \in[0,1]$, captures the extent of technological spillovers. Both these parameters are industry-specific and exogenously determined by technology. Note that the above function implies that there are decreasing returns to R\&D. This assumption, which is very common in this class of models, is necessary if the second-order condition for an interior maximum in the R\&D stage is to be satisfied. ${ }^{2}$ Apart from that assumption, the particular form of the R\&D cost function is not crucial: for instance, none of the results reported below changes if one uses the function $u_{i}=\varepsilon R_{i}^{1 / 3}+\varepsilon \rho R_{j}^{1 / 3}$ instead, although the model becomes analytically more complicated.

The use of the $\mathrm{R} \& \mathrm{D}$ cost function $u_{i}=\varepsilon R_{i}^{1 / 4}+\varepsilon \rho R_{j}^{1 / 4}$ to model R\&D spillovers is based on the commonly made assumption that the spillovers take place in $R \& D$

\footnotetext{
${ }^{2}$ As pointed out in Sutton (1998), the economics of this model depends only on the composite mapping from firms' fixed costs to firms' gross profits, rather than on the separate mappings of fixed costs to qualities and from qualities to gross profits. Thus the results would not change if we replaced $u$ by $u^{\gamma}, \gamma>0$, in the utility function and modified the R\&D cost function accordingly so that the second-order condition for an interior maximum in the R\&D stage were always satisfied. Under this more general formulation, both gross profit and R\&D cost could be either concave or convex in $u$, although gross profit would still need to be concave in R\&D cost.
} 
outcomes. An alternative approach is to regard spillovers as taking place in $R \& D$ inputs or expenditures (see Amir 2000 for a comparison of the two approaches). To check whether the results of the present paper are robust to the choice of approach to modelling spillovers I also analysed a version of the model using the alternative R\&D cost function $u_{i}=\varepsilon\left(R_{i}+\rho R_{j}\right)^{1 / 4}$. The results were very similar to those reported below: none of the propositions proved in the paper are affected by the choice of R\&D cost function.

A symmetric subgame-perfect equilibrium in pure strategies in the two-stage game can be easily computed. In the next two sections this equilibrium is characterised first for the quantity-setting (Cournot) case and then for the price-setting (Bertrand) case.

\section{Cournot Equilibrium.}

When firms set quantities in the final-stage subgame, firm $i$ chooses $x_{i}$ to maximise

$$
\pi_{i}=S\left(p_{i}-c\right) x_{i}=S\left(1-\frac{2 x_{i}}{u_{i}^{2}}-\frac{\sigma x_{j}}{u_{i} u_{j}}-c\right) x_{i}, \quad i=1,2, \quad j \neq i
$$

in the regions where prices and quantities are positive. In the Cournot-Nash equilibrium we have

$$
x_{i}{ }^{C}=\frac{(1-c) u_{i}\left(4 u_{i}-\sigma u_{j}\right)}{(4-\sigma)(4+\sigma)}, \quad \pi_{i}{ }^{C}=\frac{2 S(1-c)^{2}\left(4 u_{i}-\sigma u_{j}\right)^{2}}{(4-\sigma)^{2}(4+\sigma)^{2}}, \quad i=1,2, \quad j \neq i
$$

in the region of quality spaces where $x_{i}$ and $x_{j}$ are both positive, i.e. for $u_{i} / u_{j}<4 / \sigma, i=$ $1,2, j \neq i$. Note that if this condition did not hold, then one of the two firms (specifically, the low-quality one) would have zero sales and the other would make

\footnotetext{
${ }^{3}$ I am indebted to a referee for suggesting this alternative R\&D cost function.
} 
monopoly profit $\pi_{M}=S(1-c)^{2} u^{2} / 8$. However, this case is not relevant as a potential equilibrium of the game: for one thing, a firm would never choose a level of R\&D that resulted in zero output, since it would then be making negative net profit.

At stage 1 each firm chooses a quality level to maximise net profit $\pi-R$, given the choice of the other firm. The first-order condition for firm $i$ is

$$
\frac{d \pi_{i}^{C}}{d R_{i}}=\frac{\partial \pi_{i}^{C}}{\partial u_{i}} \frac{\partial u_{i}}{\partial R_{i}}+\frac{\partial \pi_{i}^{C}}{\partial u_{j}} \frac{\partial u_{j}}{\partial R_{i}}=1, \quad i=1,2, \quad j \neq i
$$

i.e. each firm spends on $R \& D$ up to the point where the cost of an extra unit of $R \& D$ is equal to the profit it creates in the final stage of the game. Substituting the expressions for the various partial derivatives in (5), setting $u_{i}=\varepsilon R_{i}^{1 / 4}+\varepsilon \rho R_{j}^{1 / 4}$, and solving for the (symmetric) equilibrium, we obtain

$$
u_{C}=\varepsilon(1+\rho) R_{C}^{1 / 4}=\frac{S^{1 / 2}(1-c) \varepsilon^{2}(4-\rho \sigma)^{1 / 2}(1+\rho)^{3 / 2}}{(4-\sigma)^{1 / 2}(4+\sigma)} .
$$

The second-order condition for an interior maximum at the symmetric equilibrium is given by $\partial^{2} \pi_{i}^{C} / \partial R_{i}{ }^{2}<0$ when evaluated at $u_{i}=u_{j}=u_{C}$. After some straightforward manipulations this gives

$$
\frac{-S(1-c)^{2} \varepsilon^{2}[8-3 \sigma+2 \rho(6-\sigma)] R_{C}^{-3 / 2}}{4(4-\sigma)(4+\sigma)^{2}}<0
$$

which is always true.

To compute the equilibrium price and quantity we set $u_{i}=u_{j}=u_{C}$ in (4). We obtain:

$$
x_{C}=\frac{(1-c) u_{C}^{2}}{4+\sigma}, \quad p_{C}=c+\frac{2(1-c)}{4+\sigma} \text {. }
$$


Finally, equilibrium consumer surplus, firms' profits (net of R\&D costs) and total welfare are, respectively, given as

$$
\begin{aligned}
& C S_{C}=2 S\left(x_{C}-\frac{x_{C}^{2}}{u_{C}^{2}}\right)-S \sigma \frac{x_{C}^{2}}{u_{C}^{2}}-2 S p_{C} x_{C} \\
& \Pi_{C}=2 S\left(p_{C}-c\right) x_{C}-\frac{2 u_{C}^{4}}{\varepsilon^{4}(1+\rho)^{4}}
\end{aligned}
$$

and

$$
W_{C}=2 S\left(x_{C}-\frac{x_{C}^{2}}{u_{C}^{2}}\right)-S \sigma \frac{x_{C}^{2}}{u_{C}^{2}}-2 S c x_{C}-\frac{2 u_{C}^{4}}{\varepsilon^{4}(1+\rho)^{4}} .
$$

\section{Bertrand Equilibrium.}

When firms set prices in the final-stage subgame, firm $i$ chooses $p_{i}$ to maximise

$\pi_{i}=S\left(p_{i}-c\right) x_{i}=\frac{S\left(p_{i}-c\right) u_{i}\left[2\left(1-p_{i}\right) u_{i}-\sigma\left(1-p_{j}\right) u_{j}\right]}{(2-\sigma)(2+\sigma)}, \quad i=1,2, \quad j \neq i$

in the regions where prices and quantities are positive. In the Bertrand-Nash equilibrium we have

$$
\begin{aligned}
p_{i}{ }^{B} & =c+\frac{(1-c)\left[\left(8-\sigma^{2}\right) u_{i}-2 \sigma u_{j}\right]}{(4-\sigma)(4+\sigma) u_{i}}, \quad \pi_{i}{ }^{B}=\frac{2 S(1-c)^{2}\left[\left(8-\sigma^{2}\right) u_{i}-2 \sigma u_{j}\right]^{2}}{(2-\sigma)(2+\sigma)(4-\sigma)^{2}(4+\sigma)^{2}}, \\
& i=1,2, \quad j \neq i
\end{aligned}
$$

in the region of quality spaces where $x_{i}$ and $x_{j}$ are both positive, i.e. for $u_{i} / u_{j}<$ $\left(8-\sigma^{2}\right) / 2 \sigma, i=1,2, j \neq i$. If this condition did not hold, then one of the firms would have zero sales and the other would make monopoly profit. However, such quality 
configurations can be ruled out (see the discussion in the previous section), so the above condition is always met in the two-stage game.

At stage 1 each firm chooses a quality level to maximise net profit $\pi-R$, given the choice of the other firm. The first-order condition for firm $i$ is

$$
\frac{d \pi_{i}^{B}}{d R_{i}}=\frac{\partial \pi_{i}^{B}}{\partial u_{i}} \frac{\partial u_{i}}{\partial R_{i}}+\frac{\partial \pi_{i}^{B}}{\partial u_{j}} \frac{\partial u_{j}}{\partial R_{i}}=1, \quad i=1,2, \quad j \neq i
$$

Substituting the expressions for the various partial derivatives in (12), setting $u_{i}$ $=\varepsilon R_{i}^{1 / 4}+\varepsilon \rho R_{j}^{1 / 4}$, and solving for the (symmetric) equilibrium, we obtain

$$
u_{B}=\varepsilon(1+\rho) R_{B}^{1 / 4}=\frac{S^{1 / 2}(1-c) \varepsilon^{2}\left(8-\sigma^{2}-2 \rho \sigma\right)^{1 / 2}(1+\rho)^{3 / 2}}{(4-\sigma)(4+\sigma)^{1 / 2}(2+\sigma)^{1 / 2}} .
$$

The second-order condition for an interior maximum at the symmetric equilibrium is given by $\partial^{2} \pi_{i}^{B} / \partial R_{i}^{2}<0$ when evaluated at $u_{i}=u_{j}=u_{B}$. This simplifies to

$$
\frac{-S(1-c)^{2} \varepsilon^{2}\left(8-\sigma^{2}-2 \rho \sigma\right)\left[2\left(8-3 \sigma-\sigma^{2}\right)+\rho\left(24-4 \sigma-3 \sigma^{2}\right)\right] R_{B}^{-3 / 2}}{4(4-\sigma)^{2}(4+\sigma)^{2}(2-\sigma)(2+\sigma)}<0
$$

This condition is satisfied provided $2\left(8-3 \sigma-\sigma^{2}\right)+\rho\left(24-4 \sigma-3 \sigma^{2}\right)>0$, which I assume in what follows.

The equilibrium price and quantity are derived by setting $u_{i}=u_{j}=u_{B}$ in (11). We obtain:

${ }^{4}$ It is easy to check that the second-order condition always holds if $0<\sigma<$ $(-3+\sqrt{41}) / 2$. If $(-3+\sqrt{41}) / 2 \leq \sigma<2$, then for any particular value of $\sigma$, say $\sigma_{0}$, there is a corresponding value of $\rho \in[0,1)$, say $\rho_{0}$, such that the second-order condition holds for all $\rho>\rho_{0}$. As $\sigma_{0}$ increases, so does $\rho_{0}$. 
$x_{B}=\frac{2(1-c) u_{B}^{2}}{(2+\sigma)(4-\sigma)}, \quad p_{B}=c+\frac{(1-c)(2-\sigma)}{4-\sigma}$.

The expressions for consumer surplus $C S_{B}$, firms' profits (net of R\&D costs) $\Pi_{B}$ and total welfare $W_{B}$ are similar to those under Cournot behaviour, with $p_{B}, x_{B}$ and $u_{B}$ in place of $p_{C}, x_{C}$ and $u_{C}$.

\section{Comparison.}

I begin by comparing the R\&D incentives, prices and output levels under the two regimes. From (6) and (13) we obtain:

$u_{C}^{2}-u_{B}^{2}=\frac{S(1-c)^{2} \varepsilon^{4}(1+\rho)^{4} \sigma^{3}}{(2+\sigma)(4-\sigma)^{2}(4+\sigma)^{2}}>0$

This also implies that $u_{C}>u_{B}$. Hence:

Proposition 1. Quality, and hence also R\&D expenditure, is higher under quantity competition than under price competition.

As quality increases the consumers' willingness to pay, and it is higher under Cournot than under Bertrand, it is not surprising that the ranking of equilibrium price levels in the present model is not different from the one obtained from a model without quality. In particular, (7) and (14) imply

$$
p_{C}-p_{B}=\frac{(1-c) \sigma^{2}}{(4+\sigma)(4-\sigma)}>0 .
$$

Thus:

Proposition 2. Price is higher under Cournot than under Bertrand. 
Unlike price, there is no clear ranking of the two regimes with respect to quantity produced. It is easy to check that, for any given quality, Bertrand firms produce more output than Cournot firms. However, since quality is higher under quantity competition than under price competition and high quality boosts demand, the overall ranking is ambiguous. More specifically:

Proposition 3. Output is higher in the Bertrand equilibrium than in the Cournot equilibrium if either $R \& D$ spillovers are weak or the goods are sufficiently differentiated. It is lower in the Bertrand equilibrium than in the Cournot equilibrium if $R \& D$ spillovers are strong and the goods are not too differentiated.

Proof. From equations (6), (7), (13) and (14) we obtain

$x_{B}-x_{C}=\frac{S(1-c)^{3} \varepsilon^{4}(1+\rho)^{3} \sigma^{2}\left[2\left(16-3 \sigma^{2}\right)-\rho \sigma\left(16+4 \sigma-\sigma^{2}\right)\right]}{(2+\sigma)^{2}(4-\sigma)^{3}(4+\sigma)^{3}}$.

The sign of this expression is the same as the sign of the term in brackets. Let $G$ denote that term; it can be positive or negative depending on the values of $\sigma$ and $\rho$ within the range of pairs $(\sigma, \rho)$ such that the second-order condition in the Bertrand case is satisfied and $\Pi_{B}>0$ (see below). It is easy to check that $G(\rho=0, \sigma=0)=32>$ $0, G(\rho=0, \sigma=2)=8>0$, and $G(\rho=1, \sigma=0)=32>0$. By continuity, whenever either $\rho$ is close or equal to 0 or $\sigma$ is close to 0 (or both), we have $G>0$, and hence $x_{B}$ $>x_{C}$. Moreover, $\partial G / \partial \rho=-\sigma\left(16+4 \sigma-\sigma^{2}\right)<0$ and $\partial G / \partial \sigma=-12 \sigma-\rho\left(16+8 \sigma-3 \sigma^{2}\right)$ $<0$ for all $\rho \in[0,1], \sigma \in(0,2)$; that is, $G$ decreases in both $\rho$ and $\sigma$. To complete the proof we only need to show that $x_{B}-x_{C}$ becomes negative for sufficiently large values 
of both $\rho$ and $\sigma$. This is straightforward to check; for instance, $G(\rho=0.8, \sigma=1.8)=$ $-16.1824<0$.

This result is different from the one found by Qiu (1997) in the context of a model with process $\mathrm{R} \& \mathrm{D}$; in that case, quantity sold was always higher under Bertrand than under Cournot. The reason for the difference is that, while process $\mathrm{R} \& \mathrm{D}$ reduces marginal cost, product $\mathrm{R} \& \mathrm{D}$ boosts demand. Both in the model by Qiu and in the present model Cournot firms spend more on R\&D than Bertrand firms. In the model by Qiu the direct effect of this is to lower the marginal cost of Cournot firms more than that of Bertrand firms; the main effect is therefore on profits rather than on sales. In the present model the direct effect of more R\&D by Cournot firms is that these firms experience a boost in sales stronger than the one experienced by Bertrand firms. Under certain circumstances, in particular if $R \& D$ spillovers are strong and goods are not too differentiated, this can lead to equilibrium output being higher under quantity than under price competition.

An implication of the above is that the ranking of the two regimes with respect to consumer surplus is also ambiguous:

Proposition 4. Consumer surplus is higher in the Bertrand equilibrium than in the Cournot equilibrium if either $R \& D$ spillovers are weak or the goods are sufficiently differentiated. It is lower in the Bertrand equilibrium than in the Cournot equilibrium if $R \& D$ spillovers are strong and the goods are not too differentiated.

Proof. Consumer surplus in the Cournot equilibrium is computed easily using equations (6), (7) and (8): 


$$
C S_{C}=\frac{S^{2}(1-c)^{4} \varepsilon^{4}(4-\rho \sigma)(1+\rho)^{3}(2+\sigma)}{(4-\sigma)(4+\sigma)^{4}} .
$$

Similarly, in the Bertrand equilibrium we have

$$
C S_{B}=\frac{4 S^{2}(1-c)^{4} \varepsilon^{4}\left(8-2 \rho \sigma-\sigma^{2}\right)(1+\rho)^{3}}{(2+\sigma)^{2}(4-\sigma)^{4}(4+\sigma)} .
$$

Hence

$$
C S_{B}-C S_{C}=\frac{S^{2}(1-c)^{4} \varepsilon^{4} \sigma^{2}(1+\rho)^{3} H}{(2+\sigma)^{2}(4-\sigma)^{4}(4+\sigma)^{4}}
$$

where

$$
H=4\left(128+48 \sigma-24 \sigma^{2}-7 \sigma^{3}+\sigma^{4}\right)-\rho \sigma\left(192+96 \sigma-12 \sigma^{2}-6 \sigma^{3}+\sigma^{4}\right) .
$$

The sign of $C S_{B}-C S_{C}$ is the same as the sign of $H$ : it can be positive or negative depending on the values of $\sigma$ and $\rho$ within the range of pairs $(\sigma, \rho)$ such that the second-order condition in the Bertrand case is satisfied and $\Pi_{B}>0$ (see below). We have $H(\rho=0, \sigma=0)=512>0, H(\rho=0, \sigma=2)=352>0$, and $H(\rho=1, \sigma=0)=512>$ 0 . By continuity, whenever either $\rho$ is close or equal to 0 or $\sigma$ is close to 0 (or both), we have $H>0$ and $C S_{B}>C S_{C}$. Also, $\partial H / \partial \rho=-\sigma\left(192+96 \sigma-12 \sigma^{2}-6 \sigma^{3}+\sigma^{4}\right)<$ 0 for all $\rho \in[0,1], \sigma \in(0,2)$; that is, $H$ decreases in $\rho$. On the other hand, $\partial H / \partial \sigma=$ $4\left(48-48 \sigma-21 \sigma^{2}+4 \sigma^{3}\right)-\rho\left(192+192 \sigma-36 \sigma^{2}-24 \sigma^{3}+5 \sigma^{4}\right)$, which can be positive or negative. But since $\partial^{2} H / \partial \sigma^{2}=-48\left(4-\sigma^{2}\right)-24 \sigma(7-3 \rho \sigma)-4 \rho\left(48-18 \sigma^{2}+5 \sigma^{3}\right)$ $<0$ for all $\rho \in[0,1], \sigma \in(0,2)$, we can conclude that $H$ first increases and then decreases in $\sigma$. To complete the proof, then, we only need to show that $C S_{B}-C S_{C}$ becomes negative whenever $\rho$ and $\sigma$ are both sufficiently large. This is indeed the case; for instance, $H(\rho=0.8, \sigma=1.8)=-8.79846<0$. 
Next, we compare total net profits under the two regimes. The result is not immediately obvious, since both gross industry profit and R\&D cost are higher in the Cournot equilibrium. Under quantity competition, we get from (6), (7) and (9)

$$
\Pi_{C}=\frac{2 S^{2}(1-c)^{4} \varepsilon^{4}(4-\rho \sigma)(1+\rho)^{2}[2(2-\sigma)+\rho(8-\sigma)]}{(4-\sigma)^{2}(4+\sigma)^{4}} .
$$

Similarly, under price competition we obtain

$$
\Pi_{B}=\frac{2 S^{2}(1-c)^{4} \varepsilon^{4}\left(8-2 \rho \sigma-\sigma^{2}\right)(1+\rho)^{2}\left[8-4 \sigma-\sigma^{2}+2 \rho\left(8-\sigma-\sigma^{2}\right)\right]}{(2+\sigma)^{2}(4-\sigma)^{4}(4+\sigma)^{2}},
$$

which I assume to be positive. It follows that:

$$
\Pi_{C}-\Pi_{B}=\frac{2 S^{2}(1-c)^{4} \varepsilon^{4} \sigma^{3}(1+\rho)^{3}\left[128-20 \sigma^{2}-\sigma^{3}+16 \sigma(1-\rho)+\rho\left(64-16 \sigma^{2}+\sigma^{3}\right)\right]}{(2+\sigma)^{2}(4-\sigma)^{4}(4+\sigma)^{4}}
$$

which is positive for all $\rho \in[0,1], \sigma \in(0,2)$. Hence:

Proposition 5. Profits net of $R \& D$ costs are higher under quantity competition than under price competition.

As pointed out in the Introduction, Motta (1993), who also examined a model of vertical product differentiation with fixed costs of quality improvement, found that profits are always higher in the Bertrand rather than the Cournot equilibrium, which is

\footnotetext{
${ }^{5}$ This is always the case if $0<\sigma<(-1+\sqrt{3}) / 2$. If $(-1+\sqrt{3}) / 2 \leq \sigma<2$, then for any particular value of $\sigma$, say $\sigma_{l}$, there is a corresponding value of $\rho \in[0,1)$, say $\rho_{l}$, such that $\Pi_{B}>0$ for all $\rho>\rho_{1}$. As $\sigma_{l}$ rises, so does $\rho_{l}$.
} 
the opposite of my result. However, Motta used a model that allows for quality differences between firms. In that context, firms differentiate their products (i.e. increase their quality difference), and hence 'relax' competition in the final-stage subgame, more under the price-setting regime than under the quantity-setting regime. This effect is absent from the present model, which focuses on symmetric equilibria.

Finally, we show that the overall efficiency ranking of the two regimes is ambiguous, a result which is largely a consequence of Proposition 4:

Proposition 6. Total welfare is higher in the Bertrand equilibrium than in the Cournot equilibrium if either $R \& D$ spillovers are weak or the goods are sufficiently differentiated. It is lower in the Bertrand equilibrium than in the Cournot equilibrium if $R \& D$ spillovers are strong and the goods are not too differentiated.

Proof. In the Cournot equilibrium, welfare is given by substituting equations (6) and (7) into (10):

$$
W_{C}=\frac{S^{2}(1-c)^{4} \varepsilon^{4}(4-\rho \sigma)(1+\rho)^{2}\left[16-2 \sigma-\sigma^{2}+\rho\left(24-\sigma^{2}\right)\right]}{(4-\sigma)^{2}(4+\sigma)^{4}} .
$$

For the Bertrand equilibrium we get:

$$
W_{B}=\frac{2 S^{2}(1-c)^{4} \varepsilon^{4}\left(8-2 \rho \sigma-\sigma^{2}\right)(1+\rho)^{2}\left[16-2 \sigma-\sigma^{2}+2 \rho\left(12-\sigma^{2}\right)\right]}{(2+\sigma)^{2}(4-\sigma)^{4}(4+\sigma)^{2}} .
$$

Hence:

$$
W_{B}-W_{C}=\frac{S^{2}(1-c)^{4} \varepsilon^{4} \sigma^{2}(1+\rho)^{3} J}{(2+\sigma)^{2}(4-\sigma)^{4}(4+\sigma)^{4}}
$$

where

$$
J=2\left(256-32 \sigma-64 \sigma^{2}+6 \sigma^{3}+3 \sigma^{4}\right)-\rho \sigma\left(320+64 \sigma-44 \sigma^{2}-4 \sigma^{3}+\sigma^{4}\right) .
$$


The sign of $W_{B}-W_{C}$ is the same as the sign of $J$ : it can be positive or negative depending on the values of $\sigma$ and $\rho$ within the range of pairs $(\sigma, \rho)$ such that the second-order condition in the Bertrand case is satisfied and $\Pi_{B}>0$. Now $J(\rho=0, \sigma=$ $0)=512>0, J(\rho=0, \sigma=2)=32>0$, and $J(\rho=1, \sigma=0)=512>0$. By continuity, when either $\rho$ is close or equal to 0 or $\sigma$ is close to 0 (or both), we have $J>0$, and hence $W_{B}-W_{C}>0$. Moreover, $\partial J / \partial \rho=-\sigma\left(320+64 \sigma-44 \sigma^{2}-4 \sigma^{3}+\sigma^{4}\right)<0$ and $\partial J / \partial \sigma=-(4+\sigma)\left[\left(16+60 \sigma-24 \sigma^{2}+\rho(2-\sigma)\left(40+26 \sigma-5 \sigma^{2}\right)\right]<0\right.$ for all $\rho \in[0,1]$, $\sigma \in(0,2)$; that is, $J$ decreases in both $\rho$ and $\sigma$. To complete the proof we need to show that $W_{B}-W_{C}<0$ when both $\rho$ and $\sigma$ are sufficiently large. This is straightforward to check; for instance, $J(\rho=0.8, \sigma=1.8)=-287.876<0$.

Why does the ranking of the two equilibria with respect to consumer surplus and welfare depend on the extent of R\&D spillovers and the degree of horizontal product differentiation? Recall that in the present model it is the dynamic efficiency of Cournot competition versus the static efficiency of Bertrand competition that causes the ambiguity in the overall comparison of consumer surplus and welfare in the two regimes. It is therefore not surprising that the larger the difference $u_{C}-u_{B}$, the more likely it is that the dynamic efficiency of Cournot competition dominates the static efficiency of Bertrand competition. Now it can be checked that $\partial\left(u_{C}-u_{B}\right) / \partial \rho>$ 0 and $\partial\left(u_{C}-u_{B}\right) / \partial \sigma>0 .^{6}$ In other words, not only is R\&D expenditure higher in the Cournot equilibrium than in the Bertrand equilibrium, but the difference between the

\footnotetext{
${ }^{6}$ This is true independent of the particular form of the R\&D cost function, i.e. it is true also when using the function $u_{i}=\varepsilon\left(R_{i}+\rho R_{j}\right)^{1 / 4}$.
} 
two increases as R\&D spillovers become stronger or goods become less differentiated. It follows that the larger the extent of spillovers and the less differentiated the products, the more likely it is that the dynamic efficiency of Cournot competition dominates the static efficiency of Bertrand competition.

\section{Concluding Remarks.}

The objective of this paper has been to fill a gap in the literature on the relative efficiency of quantity and price competition under product differentiation. Using a model where firms choose a level of expenditure on product R\&D prior to setting prices or quantities, I have shown that the Cournot equilibrium can be more or less efficient than the Bertrand equilibrium depending on the extent of $R \& D$ spillovers and the degree of product differentiation. The reason for the ambiguity is that firms spend more on $R \& D$ when they set quantities rather than prices. These results are consistent with those previously derived by Qiu (1997) for the case of process R\&D. A major new finding of the present paper is that, in an industry with product $R \& D$, there are circumstances where both consumer surplus and firms' profits are higher in the Cournot equilibrium than in the Bertrand equilibrium. 


\section{APPENDIX}

I analyse here an alternative version of the quality-augmented quadratic utility model, following Häckner (2000). The structure of the game is the same as before. There are $S$ identical consumers, and the utility function of each consumer takes the form

$U=u_{i} x_{i}+u_{j} x_{j}-x_{i}^{2}-x_{j}^{2}-\sigma x_{i} x_{j}+M$

where the notation is the same as in section 2. The inverse demand function of each consumer for variety $i$ is given by

$p_{i}=u_{i}-2 x_{i}-\sigma x_{j}, \quad i, j=1,2, \quad i \neq j$

in the region of quantity spaces where prices are positive. The demand function is

$x_{i}=\frac{2\left(u_{i}-p_{i}\right)-\sigma\left(u_{j}-p_{j}\right)}{(2-\sigma)(2+\sigma)}, \quad i, j=1,2, \quad i \neq j$,

in the region of prices where quantities are positive. Note that this demand function is separable in $p_{i}, u_{i}, p_{j}$ and $u_{j}$ - unlike the demand function used in the main body of the paper. Since there are $S$ consumers, firm $i$ sells quantity $S x_{i}$. The R\&D cost function is given by $u_{i}=\varepsilon R_{i}^{1 / 4}+\varepsilon \rho R_{j}^{1 / 4}$, where $\varepsilon$ and $\rho$ are as defined in section 2 .

In the quantity-setting case, the Cournot-Nash equilibrium of the final-stage subgame involves

$x_{i}{ }^{C}=\frac{4 u_{i}-\sigma u_{j}-c(4-\sigma)}{(4-\sigma)(4+\sigma)}, \pi_{i}{ }^{C}=\frac{2 S\left[4 u_{i}-\sigma u_{j}-c(4-\sigma)\right]^{2}}{(4-\sigma)^{2}(4+\sigma)^{2}}, i=1,2, \quad j \neq i$,

in the region of quality spaces where $x_{i}$ and $x_{j}$ are both positive. To obtain analytical solutions for the two-stage game, we need to set the marginal cost $c=0$. At the (symmetric) equilibrium of the two-stage game, 
$u_{C}=\varepsilon(1+\rho) R_{C}^{1 / 4}=\frac{S^{1 / 2} \varepsilon^{2}(4-\rho \sigma)^{1 / 2}(1+\rho)^{3 / 2}}{(4-\sigma)^{1 / 2}(4+\sigma)}$

(the second-order condition is always satisfied for $c=0$ ). We also obtain

$x_{C}=\frac{u_{C}}{4+\sigma}, \quad p_{C}=\frac{2 u_{C}}{4+\sigma}$

$C S_{C}=2 S u_{C} x_{C}-S(2+\sigma) x_{C}^{2}-2 S p_{C} x_{C}=\frac{S^{2} \varepsilon^{4}(4-\rho \sigma)(1+\rho)^{3}(2+\sigma)}{(4-\sigma)(4+\sigma)^{4}}$

$\Pi_{C}=2 S p_{C} x_{C}-\frac{2 u_{C}^{4}}{\varepsilon^{4}(1+\rho)^{4}}=\frac{2 S^{2} \varepsilon^{4}(4-\rho \sigma)(1+\rho)^{2}[2(2-\sigma)+\rho(8-\sigma)]}{(4-\sigma)^{2}(4+\sigma)^{4}}$

and

$W_{C}=C S_{C}+\Pi_{C}=\frac{S^{2} \varepsilon^{4}(4-\rho \sigma)(1+\rho)^{2}\left[16-2 \sigma-\sigma^{2}+\rho\left(24-\sigma^{2}\right)\right]}{(4-\sigma)^{2}(4+\sigma)^{4}}$.

In the price-setting case, the Bertrand-Nash equilibrium of the final-stage subgame involves

$$
\begin{aligned}
p_{i}^{B} & =c+\frac{\left(8-\sigma^{2}\right) u_{i}-2 \sigma u_{j}-c\left(8-2 \sigma-\sigma^{2}\right)}{(4-\sigma)(4+\sigma)}, \\
\pi_{i}^{B} & =\frac{2 S\left[\left(8-\sigma^{2}\right) u_{i}-2 \sigma u_{j}-c\left(8-2 \sigma-\sigma^{2}\right)\right]^{2}}{(2-\sigma)(2+\sigma)(4-\sigma)^{2}(4+\sigma)^{2}}, i=1,2, j \neq i,
\end{aligned}
$$

in the region of quality spaces where $x_{i}$ and $x_{j}$ are both positive. To obtain analytical solutions for the two-stage game, we need again to set $c=0$. At the (symmetric) equilibrium of the two-stage game,

$u_{B}=\varepsilon(1+\rho) R_{B}^{1 / 4}=\frac{S^{1 / 2} \varepsilon^{2}\left(8-2 \rho \sigma-\sigma^{2}\right)^{1 / 2}(1+\rho)^{3 / 2}}{(4-\sigma)(2+\sigma)^{1 / 2}(4+\sigma)^{1 / 2}}$

(the second-order condition is always satisfied for $c=0$ ). We also obtain 


$$
\begin{aligned}
& x_{B}=\frac{2 u_{B}}{(2+\sigma)(4-\sigma)}, \quad p_{B}=\frac{(2-\sigma) u_{B}}{4-\sigma} \\
& C S_{B}=\frac{4 S^{2} \varepsilon^{4}\left(8-2 \rho \sigma-\sigma^{2}\right)(1+\rho)^{3}}{(2+\sigma)^{2}(4-\sigma)^{4}(4+\sigma)} \\
& \Pi_{B}=\frac{2 S^{2} \varepsilon^{4}\left(8-2 \rho \sigma-\sigma^{2}\right)(1+\rho)^{2}\left[8-4 \sigma-\sigma^{2}+2 \rho\left(8-\sigma-\sigma^{2}\right)\right]}{(2+\sigma)^{2}(4-\sigma)^{4}(4+\sigma)^{2}}
\end{aligned}
$$

and

$$
W_{B}=\frac{2 S^{2} \varepsilon^{4}\left(8-2 \rho \sigma-\sigma^{2}\right)(1+\rho)^{2}\left[16-2 \sigma-\sigma^{2}+2 \rho\left(12-\sigma^{2}\right)\right]}{(2+\sigma)^{2}(4-\sigma)^{4}(4+\sigma)^{2}} .
$$

Comparing the two equilibria is straightforward. From (A6), (A5), (A12) and (A11) we obtain

$$
p_{C}^{2}-p_{B}^{2}=\frac{S \varepsilon^{4}(1+\rho)^{3} \sigma^{2} K}{(2+\sigma)(4+\sigma)^{4}(4-\sigma)^{4}},
$$

where

$$
K=\left(4-\sigma^{2}\right)\left(80-8 \sigma^{2}-\sigma^{3}\right)+64 \sigma(1-\rho)+16(2-\sigma)\left(6+3 \sigma-\rho \sigma^{2}\right)+2 \rho \sigma^{2}(2+\sigma)>0 .
$$

Hence $p_{C}>p_{B}$, as in proposition 2. Next, from (A6), (A5), (A12) and (A11) we obtain

$$
x_{B}^{2}-x_{C}^{2}=\frac{S \varepsilon^{4}(1+\rho)^{3} \sigma^{2} H}{(2+\sigma)^{3}(4+\sigma)^{4}(4-\sigma)^{4}},
$$

where $H$ is defined in the proof of proposition 4. Using a similar argument as the one used for proving proposition 4 , we can show that $x_{B}>x_{C}$ unless both $\rho$ and $\sigma$ are sufficiently large, in which case $x_{B}<x_{C}-$ as in proposition 3 . Finally, note that the expressions for equilibrium quality, consumer surplus, net profit and total welfare 
under both Cournot and Bertrand are identical to those obtained in the main body of the text (for $c=0$ ). It follows that propositions $1,4,5$ and 6 are also robust to the choice of demand specification. 


\section{ACKNOWLEDGMENTS}

I would like to thank Dan Kovenock (the editor) and two referees for very helpful comments on an earlier version of this paper.

\section{REFERENCES}

Amir, R., 2000, Modelling imperfectly appropriable R\&D via spillovers, International Journal of Industrial Organization, 18, 1013-1032.

Bester, H. and E. Petrakis, 1993, The incentives for cost reduction in a differentiated industry, International Journal of Industrial Organization, 11, 519-534.

Delbono, F. and V. Denicolo, 1990, R\&D investment in a symmetric and homogeneous oligopoly, International Journal of Industrial Organization, 8, 297-313.

Häckner, J., 2000, A note on price and quantity competition in differentiated oligopolies, Journal of Economic Theory, 93, 233-239.

Motta, M., 1992, Co-operative R\&D and vertical product differentiation, International Journal of Industrial Organization, 10, 643-661.

Motta, M., 1993, Endogenous quality choice: Price versus quantity competition, Journal of Industrial Economics, 41, 113-131.

Qiu, L.D., 1997, On the dynamic efficiency of Bertrand and Cournot equilibria, Journal of Economic Theory, 75, 213-229.

Singh, N. and X. Vives, 1984, Price and quantity competition in a differentiated duopoly, RAND Journal of Economics, 15, 546-554. 
Scherer, F.M. and D. Ross, 1990, Industrial Market Structure and Economic Performance (Houghton Mifflin, Boston).

Sutton, J., 1991, Sunk Costs and Market Structure (M.I.T. Press, Cambridge, MA).

Sutton, J., 1997, One smart agent, RAND Journal of Economics, 28, 605-628.

Sutton, J., 1998, Technology and Market Structure (M.I.T. Press, Cambridge, MA).

Symeonidis, G., 1999, Cartel stability in advertising-intensive and R\&D-intensive industries, Economics Letters, 62, 121-129.

Symeonidis, G., 2000, Price and non-price competition with endogenous market structure, Journal of Economics and Management Strategy, 9, 53-83.

Vives, X., 1985, On the efficiency of Bertrand and Cournot equilibria with product differentiation, Journal of Economic Theory, 36, 166-175. 